Manual

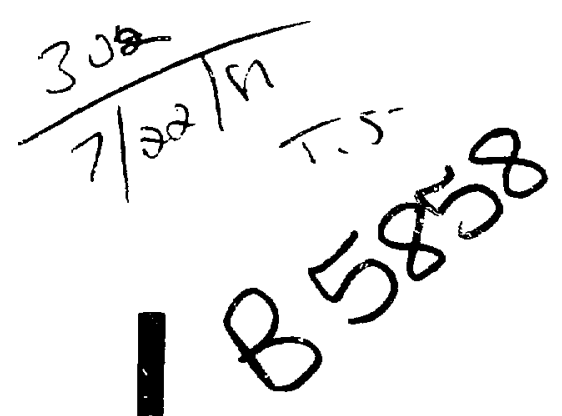

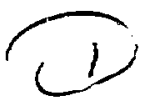

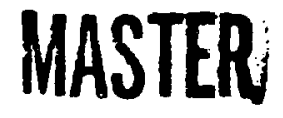

RAVEN Physics Manual

$\frac{\pi}{E}$
$\frac{0}{0}$
$\frac{\pi}{0}$
$\frac{1}{0}$
$\frac{\pi}{0}$
$\frac{1}{2}$
$\frac{1}{5}$

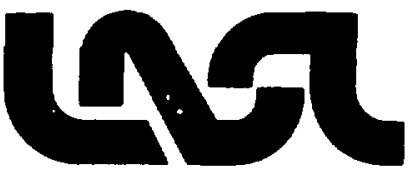




\title{
RAVEN PHYSICS MANUAL
}

by

Thomas A. Oliphant

\begin{abstract}
This document is an exposition of the physics in the RAVEN code and the numerical methods used in implementing the physics.
\end{abstract}

\section{INTRODUCTION}

The development of a one-dimensional (1-D) magnetohydrodynamics (MHD) code R.AVEN is part of a broadly based and highly flexible program being pursued at the Los Alamos National Laboratory for the study of many experimental situations. Because the purpose of this report is not a general documentation of numerical methods. mention is made only of previous work that contributed directly to the present code development. ${ }^{1.3}$

The developmental philosphy of the RAVEiv se is to broader he structure gradually to apply to more and more general situations while keeping previous simple caiculations as default options. The development is not strictly TOP.DOWN because it is impossible to ticipate all the problems that will be attacked with the code. However, it is necessary to keep the program logical and flexible. Thus, the code is not allowed simply to grow like a fungus. here is a continued effort to retain a generally TOP-DOWN code organization, althrugh the specifics of this organir inn will change as time goes on and as more experience is gained.

The overall structure will contain several options not compatible with each other, but the user should not use contradictory problem specifications and checks to avoid inconsistency. These checks are included in the code to the extent possible. For example. the calculation is formulated for a partially ionized gas with separate atomic and electronic equations for the case of hydrogen only. However. other general materials are considered provided the energies and pressures of the components are considered together in local thermodynamic equilibrium (LTE). Thus, the electronic populations are not specifically referred to in nonhydrogen materials such as copper or aluminum. The plan is to remove this limitation eventualiy. Accordingly, it is intended that a new write-up of the code be issued with any major change in code structure.

\section{MHD EQUATIONS}

In generai the materials considered are mult zomponent with different velocities. temperatures, and degrees of ionization. The approach taker in the RAVEN code is to set up a structure that handles as many options as possible, some of which may not be totally compatible with others but each of which can 
be handled within the code structure. Therefore, the fluid equations will be formulated with this objective in mind. The code attempts to include tests that disallow the use of incompatible assumptions.

One of the main influences on the formulation is the handling of the equation of state (EOS). The MAGPIE-EOS packages that are included assume LTE and a single fluid. Thus, the state variables consist of the density $\rho$, the temperature $T$, pressure $p$, and specific energy e of the total fluid. This fluid may be molecular, atomic, ionized, or a combination of these depending on the conditions. The MHD equations make no direct reference to the degree of ionization; this is taken care of internally in the transport coefficients sutrourines. At present, the SESAME2 tables are used in the same way, although a complete treatment of transport coefficients in this context has not been obtainec:

In the special case of hydrogen, it slightly more elaborate treatment has been developed. A package has been written by John D. Thomas (Los Alamos National Laboratory) that provides molecular, atomic, ionic, and electronic populations. This allows the use of the last quantity in calculating transport coefficients separately. In this package, all the components can be considered to be in LTE or the electrons can be handled separately. In the latter case. the electrons in general have a different temperature from the heavier particles. The heavier particles are assumed to have the same temperature because they equilibrate more rapidly with each other than with the electrons. In this case, two energy equations are used with temperature equilibration terms. Another LTE assumption that is relaxed is the Saha populational equilibrium between electrons. ions, and atoms. For this purpose, a rate equation is solved.

The theory is formulated to satisfy the three basic $1-D$ geometries by using index $\alpha$ in the differential operators. For example, the continuity equation for the total density $\rho$ is

$$
\frac{d \rho}{d t}+\frac{\rho}{r^{\alpha-1}} \frac{\partial}{\partial r}\left(r^{\alpha-1} u\right)=0
$$

where $\alpha$ is equal to $(1,2,3)$ for (plane, cylindrical, spherical) geometry, respectively. Because only radial motion is allowed, the radial velocity component

$$
u=\frac{d r}{d t}
$$

is the nnly one that appears.

In using MAGPIE or SESAME EOS calculations. the density $\rho$ is the only quantity needed hecause the handling of different species is done inside the subroutines. However, for the more general treatment of hydrogen. an ionization fraction $f$ is defined by

$$
n_{e}=f n_{a}
$$

where $\mathrm{n}_{\mathrm{a}}$ is the number density of atoms present whether ionized or not and whether combined into molecules or not. The ionization fraction $f$ is determined by the electron continuity equation that with the help of Eq. (3) is written in the form

$$
\frac{d f}{d t}+\frac{f}{r^{\alpha-1}} \frac{\partial}{\partial \tau}\left(r^{\alpha-1} u\right)=-\alpha f+(1-f) s
$$

The $a$ and $S$ coefficients on the right of Eq. (4) are the recombination and ionization coefficients as tabulated by Bates, Kingston, and McWhirter." This $a$ is not to be confused with the index in the differential operators. This equation enters the calculation only in the case of non-LTE hydrogen. 
The magnetic fields and currents have components only perpendicular to the radial coordinate. When the simple Ohm's law is used, the only components of the momentum equations that need be considered are the radial ones.

$$
\begin{aligned}
& n_{1} m_{1} \frac{d u_{i}}{d t}=e n_{1}\left[E_{r}+\left(u_{1} \times \underline{B}\right)_{r}\right\}-\frac{\partial p_{i}}{\partial r}+p_{i e, r} . \\
& n_{e} m_{e} \frac{d u_{e}}{d t}=-e n_{e}\left[E_{r}+\left(\underline{u}_{e} \times \underset{B}{B}\right)_{r} j-\frac{\partial p_{e}}{\partial r}+P_{e f, r} .\right.
\end{aligned}
$$

Because $m_{e} \ll m_{1}$, the inertial term in Eq. (6) is ignored and the radial electrostatic field is given by

$$
e n_{e} E_{r}=(I X B)_{r}-\frac{\partial P_{e}}{\partial r}+P_{e 1, r} .
$$

Using Eq. (7) and the charge neutrality assumption

$$
n_{e}=n_{1}
$$

allows elimination of $E_{r}$ from Eq. (5) so that

$$
D \frac{d u}{d t}=(\underline{X} X \underline{\underline{B}})_{r}-\frac{\partial p}{\partial r} .
$$

where $\rho \simeq n_{1} m_{1}$ and $u \simeq u_{1}$. The total pressure is

$$
p=p_{1}+p_{e} \cdot
$$

To take proper account of the neutral component, another contribution from the neutral momentum equation is added in; $\rho$ then includes a neutral component and the pressure becomes

$$
p=p_{a}+p_{e}
$$

where the a-species includes all atoms whether neutral, ionized, or within molecules. In the MAGPIE reduction, $p_{e}$ is removed as such and considered to be included in $p_{2}$ where, in that case. species $a$ is considered to include all particles. Note that, although the radial electrical field $E_{\mathrm{r}}$ is indeed strong, it has been eliminated from consideration and can be calculated as an edit from Eq. (7) if desired.

The energy equation for atoms is

$$
\frac{d e_{a}}{d t}+p_{a} \frac{d v}{d t}-\frac{v}{z^{\alpha-1}} \frac{\partial}{\partial r}\left(r^{\alpha-1} \kappa_{a} \frac{\partial T_{a}}{\partial r}\right)+\frac{T_{a}-T_{e}}{\tau_{e q}}=0,
$$

where

$$
v \equiv \frac{1}{0} .
$$


The pressure and energy EOS are taken to be of the form

$$
\begin{aligned}
& \mathrm{p}=\mathrm{p}\left(\mathrm{T}_{\mathrm{a}}, \mathrm{T}_{\mathrm{e}}, \cdots\right) . \\
& \mathrm{e}_{\mathrm{a}}=\varepsilon_{\mathrm{a}}\left(\mathrm{T}_{\mathrm{a}}, \cdots\right) .
\end{aligned}
$$

and

$$
e_{e}=e_{e}\left(T_{i}, v\right) .
$$

Although $e_{\mathrm{c}}$ does not appear in Eq. (11), it appears in the electron energy equation. In the MAGPIE case. $t_{e 4} \rightarrow \infty$, and $e_{a}$ includes the electron specific energy. In this case, there will be no separate electron energy equation. The first term in Eq. (11) is written

$$
\frac{d e_{a}}{d t}=e_{B, T} \frac{d T_{a}}{d t}+e_{a,:} \frac{d:}{d t}
$$

where

$$
e_{a}, T_{a}-\frac{s_{a}}{T_{a}} \mid
$$

and

$$
e_{a,}=\left.\frac{i e_{a}}{\partial{ }^{\prime}}\right|_{I_{a}} .
$$

From the first law of thermodynamics.

$$
d e_{3}=T_{a} d S_{a}-P_{a} d \because \quad \text {. }
$$

A standard thermodynamic relation is

$$
\left.\frac{d S_{a}}{d \because}\right|_{i_{a}}=\left.\frac{d p_{a}}{d T_{a}}\right|_{Y} .
$$

Thus, the first law can be written in the form

$$
e_{a_{1},}+p_{a}=T_{a} P_{a,} T_{a} .
$$

where

$$
F_{a, T_{a}} \frac{\partial p_{a}}{\partial T_{a}} \because .
$$


Note that $v$ is used instead of $v_{a}$ because $e_{a}$ is defined as the energy of atoms per total mass. The same thing will be true of the electronic component. Furthermore, because

$$
\frac{\partial \mathrm{p}_{\mathrm{a}}}{\partial \mathrm{T}_{\mathbf{a}}}=\frac{\partial \mathrm{p}}{\partial \mathrm{T}_{\mathbf{a}}}
$$

Eq. (15) can finally be written in the form

$$
e_{a}, T_{a} \frac{d I_{a}}{d t}+I_{a} P_{a} \frac{d v}{d t}-\frac{v}{r^{\alpha-1}} \frac{\partial}{\partial r}\left(r^{\alpha-1} \kappa_{a} \frac{\partial I_{a}}{\partial r}\right)+\frac{I_{a}-T_{a}}{t_{e q}}=0 .
$$

The electron energy equation is handled in a similar way.

$$
\begin{gathered}
e_{e}, T_{e} \frac{d T_{e}}{d t}+I_{e} P_{e} \frac{d v}{d t}-\frac{v}{r^{a-1}} \frac{\partial}{\partial r} \cdot r^{a-1} \times e \frac{\partial T_{e}}{\partial r} \\
+\frac{I_{e}-I_{a}}{r_{e q}}-\dot{e}_{D}+\dot{e}_{b r}=0 .
\end{gathered}
$$

where the Joule heating rate $\dot{\mathrm{e}}_{\mathrm{D}}$ and bremsstrahlung loss rate $\dot{\mathrm{e}}_{\mathrm{br}}$ are given by

$$
\dot{\mathrm{e}}_{\mathrm{D}}=\frac{1}{\rho} \mathrm{J} \cdot(7 \mathrm{~J})
$$

and

$$
\dot{e}_{b r}=\frac{P_{b r}}{a}
$$

with

$$
P_{b r}=1.757 \times 10^{-40} n_{n^{T}}
$$

The bremsstrahlung formula shown here is for an optically thin medium. The method of generalizing this approximation will be discussed in a subsequent document.

With no displacement, current Maxwell's equations are

$$
\begin{aligned}
& { }_{0} J=\underline{X} X \underline{B} . \\
& -\frac{\partial \underline{B}}{\partial t}=\underline{\nabla} \times \underline{E}, \\
& \underline{Z} \cdot \underline{B}=0 .
\end{aligned}
$$

and

$$
\underset{\sim}{\nabla}=\frac{e}{\varepsilon_{0}}\left(n_{1}-n_{e}\right) .
$$


The simple form of Ohm's law is used,

$$
\underline{E}=r_{\underline{J}}-\underline{\underline{X}} X \underset{B}{B},
$$

where $\mathrm{u}$ is given by

$$
\underline{u}=\frac{i}{\rho}\left(n_{i} m_{i} u_{1}+n_{e} m_{e} y_{e}\right)=y_{i}
$$

and $\eta$ is a tensor quantity.

In the reduction of the magnetic equations, keep in mind that all magnetic equations as well as all magnetic terms in the momentum and energy equations are only meaningful for $a=(1,2)$. In the I-D geometry, Ampere`s law, Eq. (2I), reduces to

$$
J_{0}=-\frac{1}{\nu_{0}} \cdot \frac{\partial H_{z}}{\partial r}
$$

and

$$
J_{z}=\frac{1}{r_{0} r^{x-1}} \frac{a}{o r}\left(r^{a-1} B_{B .}\right)
$$

Thus. the momentum Eq. (8) can be written as

$$
a \frac{d u}{d t}+\frac{\partial}{\partial r} z^{2}+\frac{B_{z}^{2}}{2 u_{0}}+\frac{1}{r^{2(\alpha-1)}} \frac{\partial}{d r} \cdot \frac{r^{2(\alpha-1)} B_{\frac{1}{2}}^{2}}{2 d_{0}}=0 \text {. }
$$

Faraday's law. Eq. (22), and Ohm's law. Eq. (25), are written

$$
\begin{aligned}
& \frac{\partial \mathrm{B}_{i}}{\partial t}=\frac{\Delta \mathrm{E}_{z}}{\partial \mathrm{r}} .
\end{aligned}
$$

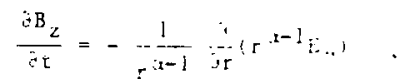

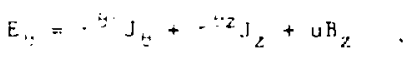

and

$$
F_{2}=i_{3}+z_{3}+z_{I_{2}}-u B
$$

For purposes of solution. the current components Eqs. (27) and (28) and the electric field components E4s. (32) and (33) are substituted into Eqs. (30) and (31) leaving two equations.

$$
\frac{\partial B_{\partial}}{\partial t}=-\frac{\partial}{\partial r} \cdot \frac{\partial z}{U_{C}} \frac{\partial B_{z}}{\partial r}+\frac{\partial}{\partial r} \frac{r^{z z}}{u_{0} r^{\alpha-1}} \frac{\partial}{\partial r}\left(r^{\alpha-1} B_{\theta}\right) !-\frac{\partial}{\partial r}\left(U B_{S}\right)
$$

and 


$$
\begin{aligned}
\frac{\partial B_{z}}{\partial t}= & \frac{1}{r^{\alpha-1}} \frac{\partial}{\partial r}\left(\frac{r^{\alpha-1} \eta \odot 0}{u_{0}} \frac{\partial B_{z}}{\partial r}\right)-\frac{1}{r^{\alpha-1}} \frac{\partial}{\partial r}\left[\frac{\eta^{\theta z}}{u_{0}} \frac{\partial}{\partial r}\left(r^{\alpha-1} B_{\theta}\right)\right] \\
& -\frac{1}{r^{\alpha-1}} \frac{\partial}{\partial r}\left(r^{\alpha-1} u B_{z}\right) .
\end{aligned}
$$

The basic set of equations to be solved includes Eqs. (4), (29), (16), (17), (34), and (35). Equations (30)-(33) are used after the solution of the basic set of equations to evaluate current and electric field components.

\section{LAGRANGIAN COORDINATES}

The position $r$ of any point moving with the fluid can be regarded as a function $r(x, t)$, where $x$ is a monutonically increasing function of the initial fluid position. Thus, any quantity f expressed as a function of space and time is now form

$$
f=f(r(x, t), t) .
$$

The space and time derivatives of $f: n$ the Lagrangian frame are obtained using the chain rule

$$
\left.\frac{\partial f}{\partial x}\right|_{t}=\left.\left.\frac{\partial f}{\partial r}\right|_{t} \frac{\partial r}{\partial x}\right|_{t}
$$

and

$$
\left.\frac{\partial f}{\partial t}\right|_{x}=\left.\left.\frac{\partial f}{\partial r}\right|_{t} \frac{\partial r}{\partial t}\right|_{x}+\left.\frac{\partial f}{\partial t}\right|_{r} .
$$

The fluid velocity $u$ at a point fixed in the fluid is given by

$$
u=\left.\frac{\dot{\partial} r}{\partial t}\right|_{x} \quad .
$$

Thus, the transformation of the time derivative, Eq. (37), becomes

$$
\left.\frac{\partial f}{\partial t}\right|_{r}=\left.\frac{\partial f}{\partial t}\right|_{x}-\left.u \frac{\partial f}{\partial r}\right|_{t} .
$$

The total time derivative is therefore

$$
\left.\frac{d}{d t} \equiv \frac{\partial}{\partial t}\right|_{r}+\left.u \frac{\partial}{\partial r}\right|_{t}=\left.\frac{\partial}{\partial t}\right|_{x} .
$$

Substituting Eqs. (36) and (40) into Eq. (1) gives

$$
\left.\frac{\partial \rho}{\partial t}\right|_{x}=-\frac{\rho}{\frac{\partial}{\partial x}\left(\frac{r^{\alpha}}{\alpha}\right)_{t}} \frac{\partial}{\partial x}\left[\frac{\partial}{\partial t}\left(\frac{r^{a}}{a}\right)_{x}\right]_{t} .
$$


Because $x$ and $t$ are independent, this can be written as

$$
\left.\frac{\partial \rho}{\partial t}\right|_{x}=-\frac{\rho}{\frac{\partial}{\partial x} \cdot \frac{r^{\alpha}}{a} \cdot t} \frac{\partial}{\partial t} \cdot \frac{\partial}{\partial x} \frac{r^{\alpha}}{\alpha} t_{t} x \cdot
$$

For this relation to be satisfied, the quantity in brackets must have the same $t$-dependence as $p(x, t)^{-1}$. Thus,

$$
\frac{\partial}{\partial x} \cdot \frac{r^{a}}{i x}=g(x) \frac{f(x, 0)}{1(x, t)} .
$$

where $g(x)$ is an arbitrarily chosen function. The relative volume or inverse compression $V$ is defined by

$$
v=\frac{v}{v_{0}}=\frac{f_{0}}{c_{0}} \text {. }
$$

where $v$ is the specific volume and the zero subscripts refer to the initial values $\rho_{0}=\rho(x, 0)$ and $v_{0}=$ $v(x, 0)$. The transformation Eq. $(4 l)$ is therefore written

$$
\frac{\partial}{\partial x}\left(\frac{r^{a}}{a}\right)=g(x) v(x, t)
$$

Because $V(x, 0)=1$, Eq. (42) is integrated over $x$ at $t=0$ to give

$$
r(x, 0)^{\alpha}=r(0,0)^{\alpha}+\alpha_{0}^{-x} g\left(x^{\prime}\right) d x^{\circ} .
$$

The simplest choice for $\mathrm{g}$ and the one that will be used in the following sections is $\mathrm{g}(\mathrm{x})=1$, in which case

$$
x=\frac{r(x, 0)^{x}-r(0,0)^{x}}{a} .
$$

This is not the only choice; for example. $g(x)=x$ leads to

$$
x=r(x, 0)
$$

provided $r(0,0)$ is set to zero. The form in Eq. (43) makes $x$ proportional to the original volume of material. This might suggest that mass rather than original volume be used as the Lagrangian coordinate. However, this is less good in situations where there is creation or destruction of the fluid component under consideration. Although this does not occur in the present model, future extensions of the theory will require it.

With $g(x)=1$, Eq. (42) becomes

$$
\frac{j}{i x} \frac{r^{1}}{x_{t}}=v(x, t)
$$

and the transformations from Eqs. (36) and (39) are written 


$$
\left.\frac{\partial}{\partial r}\right|_{c}=\left.\frac{r^{a-1}}{v} \frac{\partial}{\partial x}\right|_{t}
$$

and

$$
\left.\frac{\partial}{\partial t}\right|_{r}-\left.\frac{\partial}{\partial t}\right|_{x}-\left.\frac{u r^{\alpha-1}}{v} \frac{\partial}{\partial x}\right|_{t}
$$

In the remainder of the test, the subscripts that indicate the quantities held constant under differentiation will be omitted.

Application of Eqs. (45) and (46) to the relevant equations of Sec. II results in the following set of equations.

$$
\begin{aligned}
& \frac{d f}{d t}+\frac{f}{v} \frac{\partial}{\partial x}\left(r^{\alpha-1} u\right)+a f-(1-f) S=0 . \\
& \frac{d u}{d t}+\frac{r^{\alpha-1}}{\rho_{0}} \frac{\partial}{\partial x}\left(p+\frac{z_{2}^{2}}{2 u_{0}}\right)+\frac{1}{\rho_{0} r^{\alpha-1}} \frac{\partial}{\partial x}\left(\frac{I^{2(\alpha-1)} B_{\odot}^{2}}{2 u_{0}}\right)=0 . \\
& e_{a, T_{a}} \frac{d T_{a}}{d t}+\frac{T_{a} P_{a}, T_{a}}{\rho_{0}} \frac{d V}{d t}-\frac{1}{\rho_{0}} \frac{\partial}{\partial x}\left(\frac{I^{2(a-1)} \kappa_{a}}{V} \frac{\partial T_{a}}{\partial x}\right)+e_{a}, T_{a} \frac{T_{a}-T_{e}}{t_{e q}}=0 . \\
& e_{e, T_{e}} \frac{d T_{e}}{d t}+\frac{T_{e} P_{e}, T_{e}}{\rho_{0}} \frac{d V}{d t}-\frac{1}{\rho_{0}} \frac{\partial}{\partial x}\left(\frac{r^{2(\alpha-1)} K_{e}}{V} \frac{\partial T_{e}}{\partial x}\right)+e_{e}, T_{e} \frac{T_{e}-T_{1}}{t_{e q}} \\
& -e_{D}+e_{b r}=0 \text {. } \\
& J_{\odot}=-\frac{r^{\alpha-1}}{\mu_{0} V} \frac{\partial B_{2}}{\partial x} \\
& J_{z}=\frac{1}{H_{0} v} \frac{\partial}{\partial x}\left(r^{\alpha-1} B_{\theta}\right) \\
& \frac{\partial B_{\Theta}}{\partial t}=-\frac{r^{a-1}}{v} \frac{\partial}{\partial x}\left(\frac{r^{a-1} \eta^{\theta z}}{\mu_{0} v} \frac{\partial B_{z}}{\partial x}\right)+\frac{r^{\alpha-1}}{v} \frac{\partial}{\partial x}\left[\frac{\eta^{z z}}{\mu_{0} v} \frac{\partial}{\partial x}\left(r^{a-1} B_{\theta}\right)\right] \\
& +\frac{u r^{\alpha-1}}{v} \frac{\partial B_{\theta}}{\partial x}-\frac{r^{a-1}}{v} \frac{\partial}{\partial x}\left(u B_{\theta}\right) . \\
& \frac{\partial B_{z}}{\partial t}=\frac{1}{V} \frac{\partial}{\partial x}\left[\frac{r^{2(\alpha-1)} \eta_{\eta} \theta \theta}{\mu_{0} V} \frac{\partial B_{z}}{\partial x}\right\}-\frac{1}{V} \frac{\partial}{\partial x}\left[\frac{r^{\alpha-1} \eta^{\theta z}}{\mu_{0} V} \frac{\partial}{\partial x}\left(r^{\alpha-1_{B}}\right)\right] \\
& +\frac{u r^{\alpha-1}}{V} \frac{\partial B_{2}}{\partial x}-\frac{1}{V} \frac{\partial}{\partial x}\left(r^{\alpha-1} u B_{2}\right) .
\end{aligned}
$$


The transiormation to Lagrangian coordinates was set up to satisfy the continuity equation. Therefore, the latter needs no further explicit consideration.

Equations (53) and (54) can be put into flux-conserving form in the following way. Differentiation of Eq. (44) with respect to time gives

$$
\frac{\partial V}{\partial t}=\frac{\sum}{d x}\left(r^{i-1} u\right) .
$$

By means of Eq. (55), the velocity terms as such are eliminated from Eqs. (53) and (54), giving

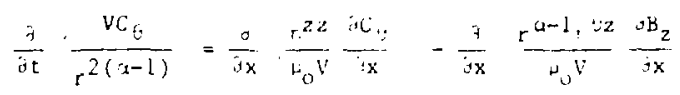

and

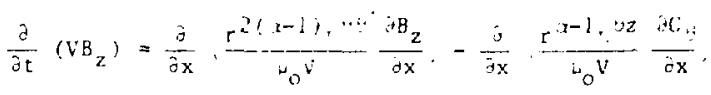

where

$$
C_{5}-r^{\alpha-B_{B}} .
$$

The motion of the medium is taken into account through the time ependence of $V(x . t)$. The basic set of partial differential equations (PDE) to be solved includes Eqs. (47) - (50) and Eqs. (56) and (57). Equations (51) and (52) are used after the fact to express the current components. The quantity $\mathrm{C}_{6}$ is constant in a vacuum region and is therefore better to use than $B_{\theta}$ in the nurrerical treatment.

\section{FINITE DIFFERENCE EQUATIONS}

The domain of solution in $r$ will always be finite $a \leqslant r \leqslant b$, and for $\alpha=2,3$, it is required that $a \geqslant 0$. For $\alpha=1$, the physical space is infinite. but is treated on a per unit area basis for $\alpha=: 1$ and on a per unit length basis for $a=2$. In the latter case. the physical domain is as illustrated in Fig. 1. The Lagrangian variable $x$ is defined nver the domain $x_{a} \leqslant x \leqslant x_{b}$, where $x_{a}=a^{a} / a$ and $x_{b}=b^{\alpha} / a$. The variable $x$ is made discrete by choosing a finite set of values $x_{1}$ monotonically increasing with the integer index $i$ where $0 \leqslant i \leqslant I$. The extreme values of $x_{1}$ are made to coincide with the boundaries so that $x_{\mathrm{c}}=\mathrm{x}_{\mathrm{a}}$ and $\mathrm{x}_{1}=\mathrm{x}_{\mathrm{b}}$. Thus a set of cells is formed as shown in Fig. 2 with each index corresponding to a cell edge. As will be seen below, many quantities will be defined at cell centers and will be labeled by a half-integer as is also shown in Fig. 2. As indicated in the figure, the cells need not be of uniform size. The discrete time variable $t^{n}$ is a monotonically increasing function of the integral index $n$.

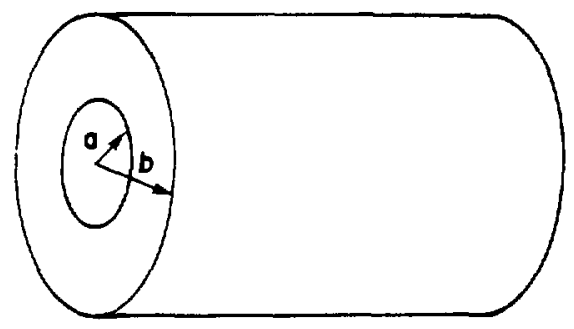

Fig. 1. The basic cylindrical system. 


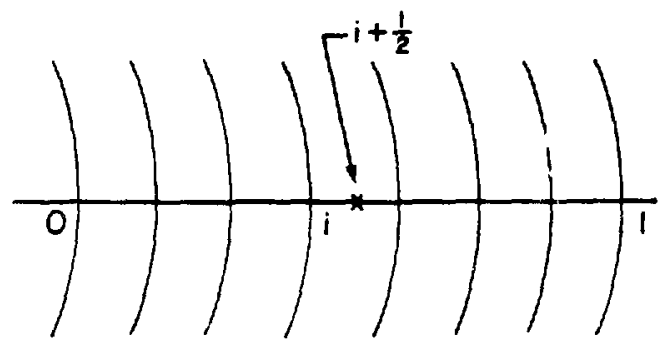

Fig. 2. The radial mesh.

The discrete position $r_{i}^{n}$ of the fluid particle is written $r_{1}^{n}\left(x_{i}, t^{n}\right)$. In the following sections, indexing subscripts will refer to Lagrangian position and indexing superscripts to time, as in the above example.

The velocity of a cell edge or "mass point" is given by the difference form of Eq. (38).

$$
u_{i}^{n-1 / 2}=\frac{r^{n}-r^{n-1}}{\Delta t^{n-1 / 2}}
$$

where

$$
\Delta t^{n-1 / 2} \equiv t^{n}-t^{n-1} \text {. }
$$

The value of $V$ for a given cell center is obtained by using the difference form of Eq. (44),

$$
v_{i+1 / 2}^{n}=\frac{\left(r_{i+1}^{n}\right)^{\alpha}-\left(r_{i}^{n}\right)^{\alpha}}{\alpha \Delta x_{i+1 / 2}}
$$

where

$$
\Delta x_{i+1 / 2} \equiv x_{i+1}-x_{i}
$$

The quantity $V_{1+1 / 2}^{n}$ is defined at a cell center as most other quantities will be. The integer or half-integer nature of the subscript will always indicate where the quantity in question is defined. When a quantity is required in a position other than where it is defined to be, it will be so indicated with a bar representing an average.

For a quantity $Q$ normally defined at a cell edge, the cell center value is given by

$$
Q_{i+1 / 2}^{n}=\frac{Q_{i+1}^{n}+Q_{i}^{n}}{2}
$$

For a quantity $\mathrm{Q}$ normally defined at a cell center, the cell edge value is given by

$$
Q_{i}^{n}=\frac{\Delta x_{i+1 / 2} Q_{i-1 / 2}^{n}+\Delta x_{i-1 / 2} Q_{i+1 / 2}^{n}}{Q_{i+1 / 2}^{n}+Q_{i-1 / 2}^{n}} .
$$

The implicit difference equations are as follows. Equation (47) becomes 


$$
\begin{aligned}
& \frac{f_{1+1 / 2}^{n}-f_{1+1 / 2}^{n-1}}{A r^{n-1 / 2}}+\frac{f_{i+1 / 2}^{n}}{v_{1+1 / 2}^{n+1}} \frac{\left(\left(r_{1+1}^{n}\right)^{n-1} u_{1+1}^{n-(1 / 2}-\left(r_{1}^{n}\right)^{(x-1} u_{1}^{n-1 / 2}\right)}{2 x_{1+1 / 2}}
\end{aligned}
$$

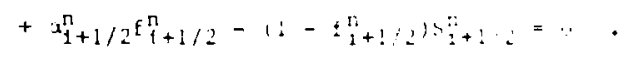

The momentum Eq. $(4 \varepsilon)$ is written

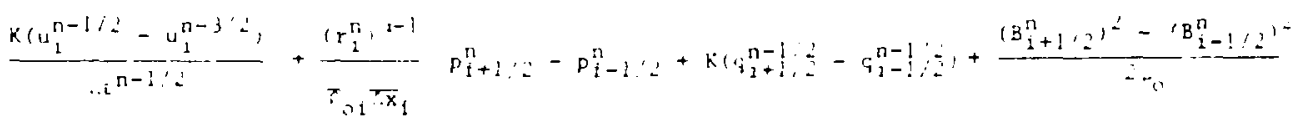

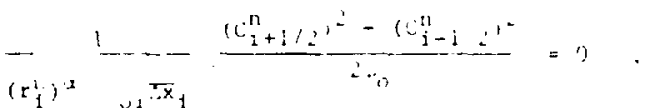

where the arificial viscosity $q_{1.1}^{n} 1 ;$ is given by

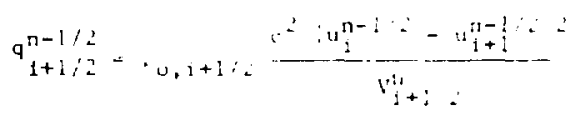

for $u_{1}^{n} \geqslant u_{i, 1}^{n}$ and zero cherwise. where $c$ is a constant of order unity. For a forced quasi steady pressure balance. $K$ is set to zero. For a dynamic calculation. $K$ is set to unity.

The implicit difference approximations to Eqs. (49) and (50) are

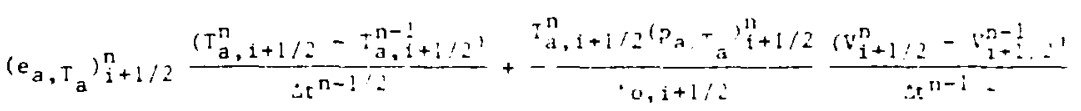

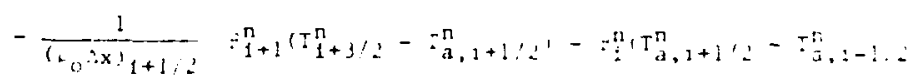

$$
\begin{aligned}
& +\left(e_{a}, T_{a}\right)_{i+1 / 2}^{n} \frac{T_{a, 1+1}^{n}-T_{r, i+1}^{n}=i}{t_{e q, 2+1}^{n} \cdot 2}=i
\end{aligned}
$$

and

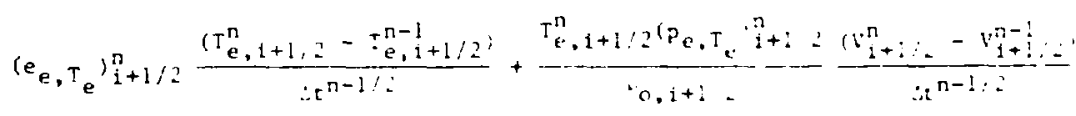

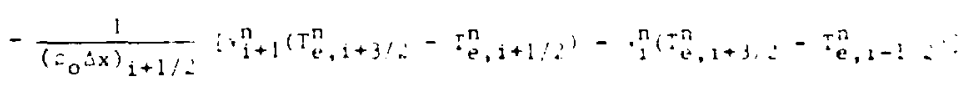

$$
\begin{aligned}
& +\left(e_{e}, T_{e}\right)_{1+1 / 2}^{n} \frac{T_{e}^{n}, 1+1 / 2-T_{a, 1+1 / 2}^{n}}{\tau_{e q, 1+1 /:}^{n}}-\left(\dot{e}_{D}\right)_{1+1 ; 2}^{n}+\left(\dot{e}_{b r}\right)_{1+1 / 2}^{n}=0 .
\end{aligned}
$$


where

$$
B_{1}^{n}=\frac{\left(r_{1}^{n}\right)^{2(a-1)}}{2}\left[\left(\frac{c_{a}}{v \Delta x}\right)_{1+1 / 2}^{n}+\left(\frac{\alpha a}{v \Delta x}\right)_{1-1 / 2}^{n} j\right.
$$

and

$$
r_{1}^{n}=\frac{\left(r_{1}^{n}\right)^{2(a-1)}}{2}\left[\left(\frac{x_{e}}{v \Delta x}\right)_{1+1 / 2}^{n}+\left(\frac{x_{e}}{v \Delta x}\right)_{1-1 / 2}^{n}\right] .
$$

The implicit difference approximations to Eggs. (56) and (57) are

$$
\begin{aligned}
& \frac{v_{1+1 / 2}^{n} C_{1+1 / 2}^{n}}{\left(r_{1+1 / r^{n}}^{n}\right)^{2(a-1)}}-\frac{v_{1+1 / 2}^{n-1} C_{1+1 / 2}^{a-1}}{\left(\widetilde{r}_{1+1 / 2}^{n-1}\right)^{2(a-1)}} ; \frac{1}{\Delta t^{n-1 / 2}} \\
& -\frac{1}{u_{0} \Delta x_{1+1 / 2}} !\left(a^{z z}\right)_{1+1}^{n}\left(c_{1+3 / 2}^{n}-c_{1+1 / 2}^{n}\right)-\left(a^{2 z}\right)_{1}^{n}\left(c_{1+1 / 2}^{n}-c_{1-1 / 2}^{n}\right) ! \\
& +\frac{1}{u_{0}^{\Delta x_{1+1} / 2}}\left\{\left(a^{\theta z}\right)_{i+1}^{n}\left(B_{1+3 / 2}^{n}-B_{1+1 / 2}^{n}\right)-\left(a^{\theta z}\right)_{1}^{n}\left(B_{1+1 / 2}^{n}-B_{1-1 / 2}^{n}\right) ;=0\right.
\end{aligned}
$$

and

$$
\begin{aligned}
& \frac{v_{i+1 / 2}^{\mathrm{n}} \mathrm{B}_{1+1 / 2}^{\mathrm{n}}-v_{1+1 / 2}^{\mathrm{n}-1} \mathrm{~B}_{1+1 / 2}^{\mathrm{n}-1}}{\Delta \mathrm{c}^{\mathrm{n}-1 / 2}} \\
& -\frac{1}{u_{0}^{\Delta x_{1}+1 / 2}}\left(\left(a^{\theta \theta}\right)_{1+1}^{n}\left(B_{1+3 / 2}^{n}-B_{1+1 / 2}^{n}\right)-\left(a^{\theta \bar{\theta}}\right)_{1}^{n}\left(B_{1+1 / 2}^{n}-B_{1-1 / 2}^{n}\right) !\right. \\
& +\frac{1}{u_{0} \Delta x_{i+1 / 2}} \vdots\left(a^{\theta z}\right)_{i+1}^{n}\left(c_{i+3 / 2}^{n}-c_{1+1 / 2}^{n}\right)-\left(a^{\theta z}\right)_{1}^{n}\left(c_{i+1 / 2}^{n}-c_{1-1 / 2}^{n}\right) ; \cdot 0 .
\end{aligned}
$$

where

$$
\begin{aligned}
& \left(a^{\theta \theta}\right)_{1}^{n}=\frac{\left(r_{1}^{n}\right)^{2(a-1)}}{2}\left[\frac{\left(n^{\theta \theta}\right)_{1+1 / 2}^{n}}{v_{1+i / 2}^{n} \Delta x_{1+1 / 2}}+\frac{\left(n^{\theta \theta}\right)_{1-1 / 2}^{n}}{v_{1-1 / 2}^{n} \Delta x_{1-1 / 2}}\right], \\
& \left(a^{22}\right)_{1}^{n}=\frac{1}{2}\left[\frac{\left(n^{22}\right)_{1+1 / 2}^{n}}{v_{1+1 / 2}^{n} \Delta x_{1+1 / 2}}+\frac{\left(n^{22}\right)_{1-1 / 2}^{n}}{v_{1-1 / 2}^{n} \Delta x_{1-1 / 2}}\right],
\end{aligned}
$$

and

13 


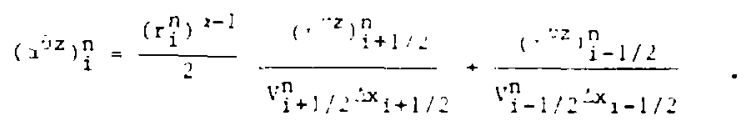

\section{SOLUTION ALGORITHM}

The solution of the difference equations of Sec. IV is accomplished by splitting off all the diffusive terms and some rate terms to obtain a truncated system that is solved implicity by Newton-Raphson iteration. This partial solution is then used in conjunction with the previously deleted terms to complete the solution. This splitting is very much like the implicit contirnous-fluid Eulerian (ICE) technique developed by Harlow and Amsden. The intermediate solution is accordingly labeled by a tilde. The truncated sy'stem is summarized as follows.

$$
\begin{aligned}
& \tilde{u}_{i}=\frac{z_{1}-r_{1}^{n-1}}{\therefore t^{n-1}-2} .
\end{aligned}
$$

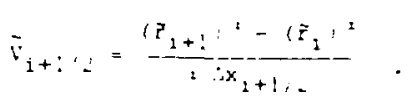

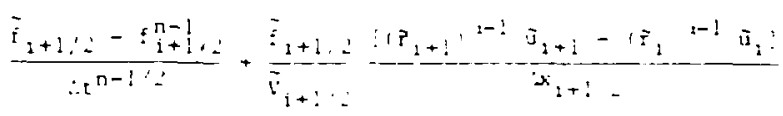

$$
\begin{aligned}
& +i_{1}+1=\bar{z}_{1+2}+1,-\bar{f}_{1+1}-\bar{z}_{1+2}==\text {. }
\end{aligned}
$$

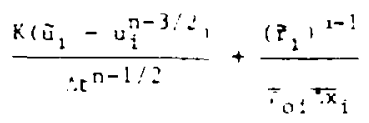

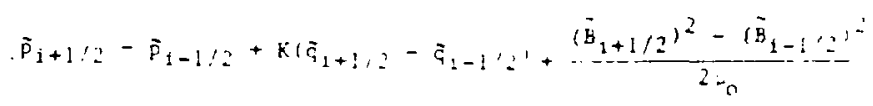

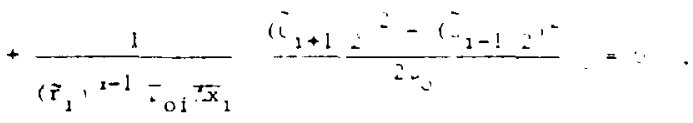

$$
\begin{aligned}
& \tilde{q}_{1+1 i}=\ldots i+1.2 \frac{c^{2} \tilde{u}_{i}-\tilde{u}_{1+i} i^{i}}{i_{i+1}} .
\end{aligned}
$$

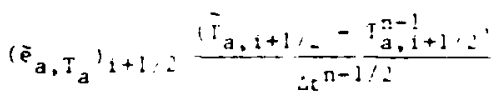

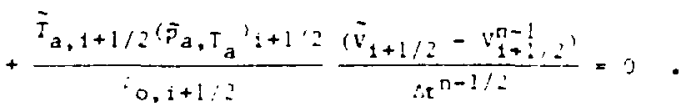




$$
\begin{aligned}
& \left(\tilde{e}_{e}, T_{e}\right)_{1+1 / 2} \frac{\left(\tilde{T}_{\left.e, i+1 / 2-T_{e, i+1 / 2}^{n-1}\right)}\right.}{\Delta e^{n-1 / 2}} \\
& +\frac{\bar{\tau}_{e, 1+1 / 2}\left(\overline{\mathrm{p}}_{\mathrm{e}}, \mathrm{T}_{\mathrm{e}}\right)_{t+1 / 2}}{\tilde{O}_{0,1+1 / 2}} \frac{\left(\overline{\mathrm{v}}_{1+1 / 2}-v_{i+1 / 2}^{\mathrm{n}-1}\right)}{\Delta \mathrm{e}^{\mathrm{n}-1 / 2}}=0 . \\
& \frac{\vec{v}_{1+1 / 2} \tilde{c}_{1+1 / 2}}{\left(\bar{r}_{i+1 / 2}\right)^{2(a-1)}}=\frac{v_{1+1 / 2}^{n-1} c_{1+1 / 2}^{n-1}}{\left(\bar{r}_{1+1 / 2}^{n-1}\right) 2(a-1)} \text {. } \\
& \bar{v}_{1+1 / 2} \tilde{B}_{1+1 / 2}=v_{1+1 / 2}^{n-1} B_{1+1 / 2}^{n-1} .
\end{aligned}
$$

This system can be reduced to a system involving strictly radial positions of the form

$$
t_{1}\left(\vec{P}_{1-1}, \vec{r}_{1}, \vec{r}_{1+1}\right)=0
$$

by the following chain of substitutions. First. $\tilde{u}_{1}$ and $\tilde{\nabla}_{1+1 / 2}$ are eliminated from the remainder of the equations by means of Eqs. (75)-(77). Then $\bar{q}_{1+1 / 2}$ is eliminated by Eq. (79). Further, $\hat{T}_{0,1+1 / 2}, \hat{T}_{e .1<1 / 2}$. $\tilde{\mathrm{B}}_{1.12}$. and $\bar{C}_{1+12}$ are eliminated by means of Eqs. (80)-(83). This leaves the momentum Eq. (78), which is now in the form of Eq. (84). This substitution procedure is not made aigebraically, but step by step numerically.

The Newton.Raphson system for solving the system Eq. (84) is

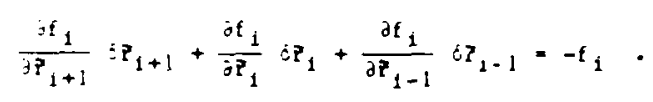

The derivatives in Eq. (85) are best evaluated numerically by, for example.

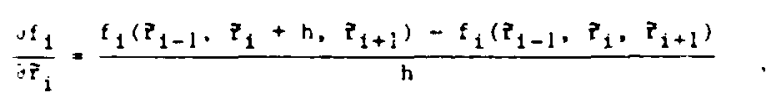

with $\mathrm{h}$ some nominal deviation. The other derivatives are given by similar expressions.

Once the intermediate solution is found, it is generally adequate though not necessary to solve the resulting system linearly. The resulting linear system for the variables at $\mathbf{~}^{\mathbf{n}}$ is given as follows.

$$
\begin{aligned}
& \left(\mathrm{B}_{a, T_{a}}\right)_{1+1 / 2} \frac{\left(T_{a, 1+1 / 2}^{n}-T_{a, 1+1 / 2}^{n-1}\right)}{\Delta t^{n-1 / 2}}+\frac{T_{a, 1+1 / 2}^{n}\left(\hat{p}_{a, T_{a}}\right)_{i+1 / 2}}{D_{0,1+1 / 2}} \frac{\left(V_{i+1 / 2}^{n}-v_{i+1 / 2}^{n-1}\right)}{\Delta t^{n-1 / 2}} \\
& -\frac{1}{\left(D_{0} \Delta x\right)_{1+1 / 2}}\left[\dot{B}_{1+1}\left(T_{a, 1+3 / 2}^{n}-r_{a, 1+1 / 2}^{n}\right)-\dot{B}_{i}\left(T_{a, i+1 / 2}^{n}-T_{a, 1-1 / 2}^{n}\right)\right] \\
& +\frac{T_{a, 1+1 / 2}^{n}-T_{e, 1+1 / 2}^{n}}{t_{e q, 1+1 / 2}}=0
\end{aligned}
$$




$$
\begin{aligned}
& \left(\bar{e}_{e, T}\right)_{1+1 / 2} \frac{\left(T_{e}^{n}, i+1 / 2-T_{e, 1+1 / 2}^{n-1}\right)}{\Delta t^{n-i} / 2}+\frac{T_{e, i+1 / 2}^{n}\left(\tilde{p}_{e}, T_{e}\right)_{i+1 / 2}}{\rho_{0,1+1 / 2}} \frac{\left(v_{i+1 / 2}^{n}-v^{n-1} i+1 / 2\right)}{\Delta t^{n-1 / 2}} \\
& -\frac{1}{\left(D_{0} \Delta x_{1+1} / 2\right)} \cdot \gamma_{1+1}\left(T_{e, 1+3 / 2}^{n}-T_{e, 1+1 / 2}^{n}\right)-\bar{\gamma}_{i}\left(T_{e, i+1 / 2}^{n}-T_{e, i+1 / 2}^{n}\right) \\
& +\frac{T_{e, 1+1 / 2}^{n}-T_{a, i+1 / 2}^{n}}{\tau_{e q, 1+1 / 2}^{n}}+\left(\dot{e}_{D}\right)_{1+1 / 2}^{n}-\left(\dot{e}_{b r}\right) \eta_{i+1 / 2}^{n}=0 \\
& \left(\frac{v_{1+1 / 2}^{n} c_{1+1 / 2}^{n}}{\left(\bar{r}_{i+1 / 2}^{n}\right)^{2(\alpha-1)}}-\frac{v_{i+1 / 2}^{n-1} c_{1+1 / 2}^{n-1}}{\left(r_{i+1 / 2}^{n-1}\right)^{2(\alpha-1)}} \cdot \frac{1}{\Delta t^{n-1 / 2}}\right. \\
& -\frac{1}{w_{0} \Delta x_{i+1 / 2}} i\left(z^{z z}\right)_{i+1}\left(c_{i+3 / 2}^{n}-C_{i+1 / 2}^{n}\right)-\left(z^{z z}\right)_{i}\left(c_{i+1 / 2}^{n}-c_{i-1 / 2}^{n}\right) . \\
& +\frac{1}{u_{0} \Delta x_{1+1} / 2}:\left(z^{\theta z}\right)_{1+1}\left(B_{i+3 / 2}^{n}-B_{i+1 / 2}^{n}\right)-\left(a^{\theta z}\right)_{1}\left(B_{i+1 / 2}^{n}-B_{i-1 / 2}^{n}\right)_{j}=0 \text {. } \\
& \frac{v_{i+1 / 2}^{n} B_{i+1 / 2}^{n}-v_{1+1 / 2}^{n-1} B_{1+1 / 2}^{n-1}}{\Delta t^{n-1 / 2}} \\
& -\frac{1}{\omega_{0} \Delta x_{1+1 / 2}}\left\{\left(z^{\theta \theta}\right)_{1+1}\left(B_{1+3 / 2}^{n}-B_{1+1 / 2}^{n}\right)-\left(z^{\theta \theta}\right)_{1}\left(B_{1+1 / 2}^{n}-B_{1-1 / 2}^{n}\right)\right\} \\
& +\frac{1}{\omega_{0} \Delta x_{1+1 / 2}}\left(\left(a^{\theta z}\right)_{1+1}\left(c_{1+3 / 2}^{n}-c_{1+1 / 2}^{n}\right)-\left(a^{\theta z}\right)_{1}\left(c_{1+1 / 2}^{n}-c_{1-1 / 2}^{n}\right) \vdots=0 .\right.
\end{aligned}
$$

The only difference between Eqs. (87) - (90) and the corresponding squations of Sec. IV is that some of the quantities are evaluated using tilde quantities. Thus, this set of equations is linearized. This completes the numerical solution.

\section{CIRCUIT COUPLING}

The self-consistent coupling of an external circuit to an interior point is best illustrated in terms of a pure Z-pinch (Fig. 3) or a pure $\theta$-pinch (Fig. 4). Although the couplings are illustrated individually, they can be combined to form a mixed pinch with no changes in the coupling cell equations.

Because the two cases are handled in a similar way, they are treated together. The entry and return current paths are assumed to enclose a single cell. As will be seen below, the coupling is achieved by merely altering the diference equations for the coupling cell.

The rate of increase of fux in the coupling cell is given by Faraday's law.

$$
-\frac{\partial}{\partial \tau} j \mathrm{~B} \cdot \mathrm{dS}=\phi \mathrm{E} \cdot \mathrm{dS} .
$$




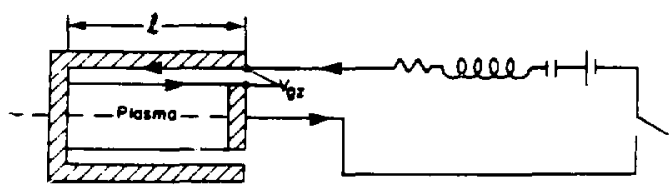

Fig. 3. The $Z$-pinch coupling circuit.

Fig. 4. The $\theta$-pinch coupling circuit.

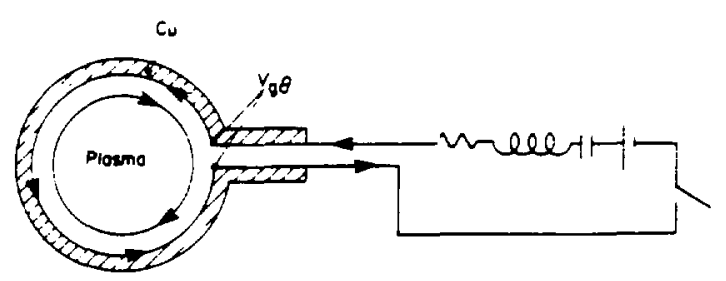

Evaluating the integrals for both cases gives

$$
-\frac{\partial}{\partial t}:\left(\operatorname{\Delta rB}_{\theta}^{\prime}{ }_{i+1 / 2}^{\prime}=\ell\left(E_{z, 1+1}^{\prime}-E_{z, i}^{\prime}\right)+v_{g z}\right.
$$

and

$$
-\frac{\partial}{\partial t}\left[2 \pi r \Delta r B_{2}\right]_{1+1 / 2}=2 \pi\left(E_{\theta, 1+1}^{\prime} r_{i+1}-E_{\theta, i}^{\prime} r_{1}\right]+v_{g \theta} .
$$

These electric fields are considered to be in the Lagrangian frame. Thus, Ampere's law and Ohm's law give

$$
\frac{\partial}{\partial t}\left(\frac{v C_{E}}{r^{2}}\right)_{i+1 / 2}-\frac{1}{\Delta x_{i+1 / 2}}\left[\left(\frac{n^{22}}{u_{0} v} \frac{\partial C_{\theta}}{\partial x}\right)_{i+1}-\left(\frac{\eta^{22}}{u_{0} v} \frac{\partial C_{\theta}}{\partial x}\right)_{1}\right]-\frac{v_{g z}}{2 \Delta x_{i+1 / 2}}
$$

and

$$
\frac{\partial}{\partial t}\left(V B_{z}\right)_{1+1 / 2}-\frac{1}{\Delta x_{i+1 / 2}}\left[\left(\frac{r^{2} \eta^{\theta \theta}}{\mu_{0} V} \frac{\partial B_{z}}{\partial x}\right)_{1+1}-\left(\frac{r^{2} \eta^{\theta \theta}}{\mu_{0} V} \frac{\partial B_{z}}{\partial x}\right)_{1}\right]-\frac{v_{g \theta}}{2 \pi \Delta x_{1+1 / 2}} .
$$

These equations agree precisely with the space difference form of Eqs. (89) and (90) for the pure Z-pinch and $\theta$-pinch except for the source terms involving the gap voltages $V_{A \theta}$ and $V_{g z}$. As will be shown below, these gap voltages are eliminated in terms of the circuit terms with lumped parameters.

Elimination of the circuit current in terms of the magnetic field components yields a set of difference equations as before with the simple addition of terms at the coupling cell involving the lumped circuit parameters. In this way the MAGPIE ${ }^{2}$ code does the circuit coupling.

This coupling breaks down when the resistivity of the interna' gas is very large, as is the case when there is no appreciable ionization. In such a case, the bracketed term in Eqs. (93) or (94) almost precisely balances $V_{g z}$ or $V_{g \theta}$, respectively, and the time derivatives thus evaluated are very inaccurate because they involve small differences of large quantities. Such a situation occurs in the gas-imbedded dense Z-pinch before the laser beam has caused appreciable ionization. 
A procedure that is a little more lengthy but gets rid of the above prchlem is obtained as follows. The set of difference equations is added together over the whole mesh for Eqs. (93) and (94). Thus, the electric field terms cancel except at the outer boundaries and the resulting relations are written as

$$
R \Delta x \frac{\partial}{\partial t}\left(\frac{V C_{\theta}}{r^{2}}\right)_{i+1 / 2}+E_{z}=R_{L}^{\prime} E_{z}\left(r_{I}^{n}\right)-E_{z}\left(r_{o}^{n}\right) ;-V_{g: z}
$$

and

$$
2 \pi \Delta x \frac{\partial}{\partial t}\left(v_{B}\right)_{1+1 / 2}+E_{\theta}=2 \pi ! r_{1}^{n} E_{\theta}\left(r \frac{n}{1}\right)-r_{0}^{n} E_{\theta}\left(r_{0}^{n}\right) j-v_{g} g .
$$

where

$$
\Xi_{z}=\frac{\ell}{\Delta t^{n-1 / 2}} \sum_{j \times i} \frac{c_{j+1 / 2}^{n} v_{j+1 / 2}^{n}}{\left(\bar{r}_{j+1 / 2}^{n}\right)^{2}}-\frac{c_{j+1 / 2}^{n-i} v_{j+1 / 2}^{n-1}}{\left(\bar{r}_{j+1 / 2}^{n}\right)^{2}} ; \Delta x_{j+1 / 2}
$$

and

$$
\Xi_{g}=\frac{2 \pi}{\Delta t^{n-1 / 2}} \sum_{j \neq i} \sum_{j+1 / 2}^{n} v_{j+1 / 2}^{n}-B_{j+1 / 2}^{n-1} v_{j+1 / 2}^{n-1}, \Delta x_{j+i / 2} \text {, }
$$

where now the time differences have beer written out in the back electromotive force (emf) terms. When perfectly conducting boundaries are assumed, the electric fields are zero in Eqs. (95) and (96). When C and $B$ are specified as functions of time on either boundary, the associated electric field components in Eqs. (95) and (96) o not vanish, but they are omitted from these equations because specification of boundary values of $\mathrm{C}$ or $\mathrm{B}$ involved elimination of these field values from these equations. Again, special consideration is given when $r_{o}^{n}=0$. The $r_{0}^{n}$ factor in the relevant term of Eq. (96) causes it to vanish. There remains only the term in Eq. (95) that accounts for the field along the axis, which we denote hy $V_{m, 0}$. Thus, for computational purposes at the coupling cell, Eqs. (95) and (96) become

$$
\ell \Delta x \frac{\partial}{\partial t} i \frac{v c_{c}}{r^{2}} ;+\Xi_{z}=-v_{p l, o}-v_{g z}
$$

anci

$$
2 \pi \Delta x \frac{\partial}{\partial \tau}\left(v_{z}\right)+\equiv_{\theta}=-v_{8 \theta}
$$

where

$$
v_{p 1,0}=\ell n^{2 z}\left(r_{0}^{n}\right) J_{z}\left(r_{0}^{n}\right)
$$

The gap voltages $V_{g \theta}$ and $V_{g z}$ are given in terms of the lumped circuit parameters from Figs. 3 and 4 by

$$
v_{g}=\frac{Q}{C}-L \frac{d l}{d t}-R I+v_{b},
$$


where $V_{b}$ is the battery voltage, and the lumped parameters refer to the sircuit connected to the $\theta$-pinch or $Z$-pinch loop as the case may be. The capacitor criarge is given by

$$
Q=Q_{0}-i_{0}^{t} I d t^{\circ} .
$$

The relation Eq. (102) along with Eq. (101) is substituted into Eqs. (98) and (99) giving

$$
\varepsilon \wedge x \frac{\partial}{\partial t}\left(\frac{V C_{\theta}}{\tau^{2}}\right)+\frac{2 \pi L}{\nu_{0}} \frac{\partial C_{\theta}}{\partial t}+\frac{2 \pi}{H_{0}}\left(\frac{\partial L}{d t}+R\right) C_{\theta}-\left(\frac{Q}{C}+V_{b}+v_{p L}+\Xi_{2} ;=0\right.
$$

and

$$
2 \pi \Delta x \frac{\partial}{\partial t}\left(V B_{z}\right)+\frac{\ell L}{\mu_{0}} \frac{\partial B_{z}}{\partial t}+\frac{\ell}{\mu_{0}}\left(\frac{d L}{d t}+R\right) B_{z}-\left(\frac{Q}{C}+V_{b}+\Xi_{\theta}\right)=0
$$

where the indices of Eqs. (104) and (105) are for the coupling cell. The coupling cell difference equations become

$$
\begin{aligned}
& \frac{\Delta x_{1+1 / 2}}{\Delta t^{n-1 / 2}}\left(\frac{v_{1+1 / 2}^{n}}{\left\langle\bar{r}_{1+1 / 2}^{n}\right\}^{2}}+\frac{2 \pi L_{z}}{\ell t_{0}}\right) C_{1+1 / 2}^{n}+\frac{2 \pi}{\ell u_{0}}\left(\frac{d L_{2}}{d t}+R_{z}\right) C_{1+1 / 2}^{n} \\
& -\frac{\Delta x_{1+1 / 2}}{\Delta t^{n-1 / 2}}\left[\frac{v_{1+1 / 2}^{n-1}}{\left(\bar{r}_{1+1 / 2}^{n-1}\right)^{2}}+\frac{2 \pi L_{z}}{Q \psi_{0}}\right] C_{i+1 / 2}^{n-1}+\left(\frac{Q_{z}}{C_{z}}+v_{b, z}+v_{p 2,2}+\equiv_{2}\right)
\end{aligned}
$$

and

$$
\begin{aligned}
& \frac{\Delta x_{1+1 / 2}}{G t}\left(v_{1+1 / 2}^{n}+\frac{\ell L_{\theta}}{2 \pi \nu_{0}}\right) B_{1+1 / 2}^{n}+\frac{\ell}{L_{0}}\left(\frac{d L_{\theta}}{d t}+r_{\theta}\right) R_{1+1 / 2}^{n} \\
& =\frac{\Delta x_{1+1 / 2}}{\Delta t^{n-1 / 2}}\left(v_{i-1 / 2}^{n-1}+\frac{\ell L_{\theta}}{1 \pi \mu_{0}}\right) B_{i+1 / 2}^{n-1}+\left(\frac{Q_{\theta}}{C_{\theta}}+v_{b, 3}+\Xi_{\theta}\right) .
\end{aligned}
$$

The lumped elements are labeled according to whether they refer to the Z-pinch or $\theta$-pinch circuit.

\section{A. The Back emf Calculation}

The back emf is calculated using Faraday's law. In integrated form,

$$
-\frac{\partial f}{\partial t}=\mathscr{q} E \cdot d \underline{s} .
$$

The path integral on the right is defined to be the negative of the back emf $\Xi$. The flux is given by

$$
\Phi=\int B \cdot d \underline{B} .
$$

Thus,

$$
\equiv-\frac{\partial}{\partial t} \int \underline{B} \cdot d \underline{S} \quad
$$


In the cases of the $\theta$-pinch and $Z$-pinch, respectively, we have

$$
E_{z}=\ell \frac{\partial}{\partial t} j B_{9} d r
$$

and

$$
\Xi_{\theta}=2 \pi \frac{\partial}{\partial t} ; B_{z} r d r .
$$

Transforming to Lagrangian coordinates gives

$$
\Xi_{2}=2 \pi \frac{\partial}{\partial t} ; \frac{C_{j} \cdot r}{r^{2}} d x
$$

and

$$
\Xi_{y}=\ell \frac{\partial}{\partial t} ; B_{z} V d x .
$$

Taking the derivative inside the integral now and replacing the integral hy a sum,

$$
\Xi_{z}=\frac{i}{\Delta t} \sum_{i=1}^{l} \cdot \frac{C V}{r^{2}}{ }_{i+1 / 2}^{n}-\frac{C V}{r^{2}}{ }^{n-1} i+1 / 2 \cdot \Delta x_{i+1 / 2}
$$

and

$$
\equiv_{\theta}=\frac{2 \pi}{\Delta t} \sum_{i=1}^{I}\left[(B V)_{i+1 / 2}^{n}-\left(B V ! i+1 / 2: \Delta x_{1+1} / 2 .\right.\right.
$$

Now suppose there is some intermediate radius $i$ used to separate a region on the outside, which will act mainly like a vacuum-filled or lumped inductance. In that region, B and C are rather close to their vacuum values, which alsc equal the value at the coupling point. Thus, the lumped inductance in the outer region, when it acts as a vacuum inductance, gives an emf $i$; the form

$$
\Xi_{z \ell}=\frac{\ell}{i} \sum_{i=T}^{I}\left[\frac{C_{1+1 / 2}^{n} v_{i+1 / 2}^{n}}{\left(r_{1+1 / 2}^{n}\right)^{2}} j-\left[\frac{C_{i+1 / 2}^{n-1} v_{i+1 / 2}^{n-1}}{\left(r_{i+1 / 2}^{n-1}\right)^{2}}, j \Delta x_{i+1 / 2}\right.\right.
$$

and

$$
\Xi_{\theta \ell}=\frac{2 \pi}{\Delta t}{\underset{1}{1=T}}_{1}^{I}\left[B_{1+1 / 2}^{n} v_{1+1 / 2}^{n}-B_{i+1 / 2}^{n-1} v_{1+1 / 2}^{n-1} j \Delta x_{1+1 / 2} .\right.
$$

The analytic form for the effective lumped inductances is

$$
L_{z \ell}=\frac{\ell \mu_{0}}{2 \pi} \log \left(\frac{{ }_{1}{ }_{1}}{{ }_{T_{T}}}\right)
$$

and 


$$
L_{G \hat{l}}=\frac{\pi \omega_{O}}{l}\left(\tau_{i}^{2}-\tau \frac{2}{i}\right) .
$$

Thus, addition of the lumped inductances, Eqs. (112) and (113), to the external circuit and subtraction of Eqs. (110) and (111) from Eqs. (108) and (109) yield an equivalent physical result. In that case, the modified back emf formulas become

$$
\begin{aligned}
\Xi_{z}= & \frac{\ell}{\Delta t}\left\{\sum_{i=1}^{i}-1\left[\frac{c_{i+1 / 2}^{n} v_{i+1 / 2}^{n}}{\left(r_{i+1 / 2}^{n}\right)^{2}}\right]-\left[\frac{c_{i+1 / 2}^{n-1} v_{i+1 / 2}^{n-1}}{\left(r_{i+1 / 2}^{n}\right)^{2}}\right\} ; \Delta x / 1+1 / 2\right. \\
& +\sum_{i=1}^{1}\left\{\frac{\left(C_{i+1 / 2}^{n}-c_{i c}^{n}+1 / 2\right) v_{i+1 / 2}^{n}}{\left(r_{i+1 / 2}^{n}\right)^{2}}\right]-\left[\frac{\left(c_{i+1 / 2}^{n-1}-c_{i}^{n-1}+1 / 2\right) v_{i+1 / 2}^{n-1}}{\left(r_{i+1 / 2}^{n}\right)} ; \Delta x / i+1 / 2\right\}
\end{aligned}
$$

and

$$
\begin{aligned}
\Xi_{\theta} & =\frac{2 \pi}{\Delta t}\left\{\sum_{i=1}^{\sum_{i}-1}\left[B_{i+1 / 2}^{n} v_{i+1 / 2}^{n}-B_{i+1 / 2}^{n-1} v_{i+1 / 2}^{n-1}\right]\right. \\
& \left.+\sum_{i I}\left[\left(B_{i+1 / 2}^{n}-B_{i+1 / 2}^{n}\right) v_{i+1 / 2}^{n}-\left(B_{i+1 / 2}^{n-1}-B_{i+1 / 2}^{n-1}\right) v_{1+1 / 2}^{n-1}\right] \Delta x_{i+1 / 2}\right\} .
\end{aligned}
$$

Thus, Eqs. (114) and (115) are used to calculate the back emf in the code. When the outer region is truly a vacuum, the second summation becomes identically zero in both Eq. (114) and Eq. (115).

With the addition of the effective lumped inductances, Eqs. (106) and (107) become

$$
\begin{aligned}
& \frac{\Delta x_{i+l / 2}}{\Delta t^{n-1 / 2}}\left[\frac{v_{i+1 / 2}^{n}}{\left(T_{i+1 / 2}^{n}\right)^{2}}+\frac{2 \pi}{\ell u_{0}}\left(L_{z}+L_{z} \ell\right)\right] c_{i+1 / 2}^{n}+\frac{2 \pi}{\ell \mu_{0}}\left[\frac{d L_{z}}{d t}+\frac{d n_{z}}{d t}+R_{z}\right] c_{i+1 / 2}^{n} \\
& =\frac{\Delta x_{i}-1 / 2}{\Delta t^{n-1}}\left[\frac{v_{1+1 / 2}^{n-1}}{\left(F_{1+1 / 2}^{n-1}\right)^{2}}+\frac{2 \pi}{\ell u_{0}}\left(L_{z}+L_{z \ell}\right)\right] c_{1+1 / 2}^{n-1}+\left(\frac{Q_{2}}{c_{z}}+v_{b, z}+v_{p 1 \cdot z}+E_{z \ell}\right)
\end{aligned}
$$

and

$$
\begin{aligned}
& \frac{\Delta x_{i+1 / 2}}{\Delta t^{n-1 / 2}}\left[v_{i+1 / 2}^{n}+\frac{\ell}{2 \pi \mu_{0}}\left(L_{\theta}+L_{\theta \ell}\right)\right]+\frac{\ell}{2 \pi \mu_{0}}\left[\frac{d L_{\theta}}{d t}+\frac{d \Lambda_{\theta}}{d t}+R_{\theta}\right] B_{i+1 / 2}^{n} \\
& =\frac{\Delta x_{i+1 / 2}}{t^{n-1 / 2}}\left[v_{i+1 / 2}^{n-1}+\frac{\ell}{2 \pi \nu_{0}}\left(L_{\theta}+L_{\theta \ell}\right)\right] B_{1+1 / 2}^{n-1}+\frac{\ell}{2 \pi}\left(\frac{Q_{\theta}}{C_{\theta}}+v_{b, \theta}+\equiv_{\theta \ell}\right) .
\end{aligned}
$$

where now $\Xi_{z f}$ and $\Xi_{\theta} /$ are evaluated by Eqs. (114) and (115).

\section{B. Multiplicative Coupling}

In calculating the behavior of machines such as ZT-40, it is necessary to consider coupling by circuits with a large number of primary turns or by many parallel voltages placed at positions along the torus. Thus, some simple adjustments must be made in Eqs. (116) and (117). 
The impedances in the primary circuit appear to the pinch column as divided by the turns ratio squared, whereas the voltages appear as divided by the turns ratio $\mathrm{N}_{\mathrm{r}}$. The same thing is true of the $\theta$-pinch circuit with $N_{\theta}$. Now. if the $Z$-current is driven by voltages placed at $T_{z}$ positions along the torus. the length $\ell$ is effectively divided by $T_{2}$. Hence, Eqs. (116) and (117) become

$$
\begin{aligned}
& \frac{\Delta x_{1+1 / 2}}{\Delta t}\left[\frac{v_{1+1 / 2}^{n}}{\left(\tau_{1+1 / 2}^{n}\right)^{2}}+\frac{2 \pi T}{\ell \mu_{0}}\left(\frac{L_{z}}{N_{z}^{2}}+T_{z}\right)\right] c_{1+1 / 2}^{n}+\frac{2 \pi}{\ell \nu_{0}}\left[\frac{L_{z, t}+R_{z, t}}{N_{z}^{2}}+\tau_{z, t} .\right. \\
& =\frac{\Delta x_{1+1 / 2}}{\Delta t^{n-1 / 2}}\left[\frac{v_{1+1 / 2}^{n-1}}{\left(\bar{T}_{1+1 / 2}^{n}\right)^{2}}+\frac{2 \pi T_{z}}{f_{H_{0}}}\left(\frac{L_{z}}{N_{z}^{2}}+T_{z}\right)\right] C_{i+1 / 2}^{n}+\frac{T_{z}}{\ell}\left[\left(\frac{Q_{z}}{C_{z}}+v_{b, z} ; \frac{1}{N_{z}}+i_{z} .\right.\right.
\end{aligned}
$$

and

$$
\begin{aligned}
& \frac{\Delta x_{1+1 / 2}}{\Delta t^{n-1 / 2}}\left(v_{i+1 / 2}^{n}+\frac{\ell}{2 \pi \mu_{0}}\left(\frac{L_{\theta}}{N_{\theta}^{2}}+T_{\theta}\right)\right] B_{i+1 / 2}^{n}+\frac{\varepsilon}{2 \pi \nu_{0}}\left[\frac{L_{\theta, L}+R}{N_{\theta}^{2}}+r_{b, z} ! B_{i+1 / 2}^{n}\right.
\end{aligned}
$$

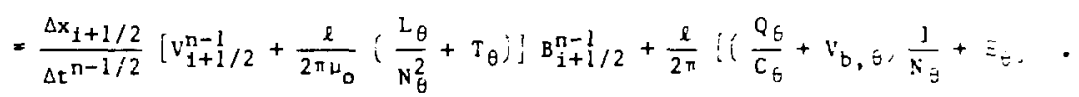

\section{Transmission Line}

In Figs. 3 and 4, the driving circuits are represented as simple LRC circuits. For some pinch calculations, this is quite adequate. However, in the high-density Z-pinch (HDZP) experiment, the main driving circuit, including a Marx bank, is connected to the load circuit, including the pinch tube, through a transmission line. The transmission line is treated using the telegrapher's equation ${ }^{7}$

$$
\frac{\partial^{2} v}{\partial x^{2}}=R^{r} v+(R C+L C) \frac{\partial v}{\partial t}+L C \frac{\partial^{2} v}{\partial t^{2}}
$$

The distributed circuit parsmeters denoted here by $R, G, C$, and $L$ are given per unit length of transmission line and are not to be confused with the lumped parameters considered in the earlier parts of this section.

The transmission line circuit is shown schematically in Fig. 5. The block $D$ represents the Marx bank and its associated circuits; the block $L$ represents the plasma load circuit including the lumped elements in

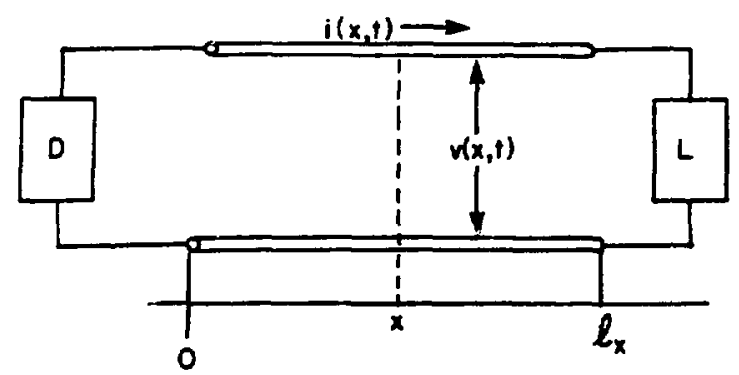

Fig. 5. The transmission line. 
Figs. 3 and 4 ; and the quantities $v(x, t)$ and $i(x, t)$ represent the voltage and current at the point $x$ along the transmission line at time $t$.

For the present treatment, it is preferable to break Eq. (120) into two first-order equations.

$$
\frac{\partial y^{\prime}}{\partial x}=-R i-L \frac{\partial i}{\partial t}
$$

and

$$
\frac{\partial 1}{\partial x}=-G \because-G \frac{\partial \because}{\partial t}
$$

This pair is rewritten as

$$
\frac{\partial \dot{z}}{\partial t}=-\frac{1}{L} i R 1+\frac{\partial v}{\partial x}
$$

and

$$
\frac{\partial j}{\partial r}=-\frac{1}{c}\left(G v+\frac{\partial i}{\partial x} ; \quad .\right.
$$

The transmission line is divided into cells along $x$ with the current given at the cell edges represented by discrete values $x_{j}$, wheze $j$ is an integer. The voltages are evaluated at cell certers where the index is half-integral. The difference analogs of Eqs. (123) and (124) are staggered both in space and tima.

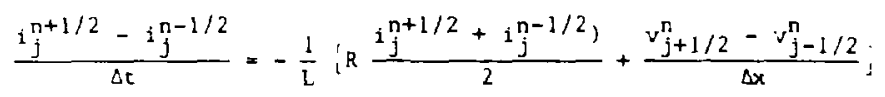

and

$$
\frac{v_{j+1 / 2}^{n+1}-v_{j+1 / 2}^{n}}{\Delta t}=-\frac{1}{c}\left[c \frac{v_{j+1 / 2}^{n+1}+v_{j+1 / 2}^{n}}{2}+\frac{1_{j-1}^{n+1 / 2}-1 j_{j}^{n-1 / 2}}{\Delta x}\right] .
$$

Equation (125) is solved for $i_{j}^{n+1 / 2}$ and Eq. (126) for $v_{j+1 / 2}^{n+1}$. Thus,

$$
i_{j}^{n+1 / 2}=\frac{\left(\frac{L}{\Delta t}-\frac{r}{2}\right) i_{j}^{n-1 / 2}-\frac{v_{j+L / 2}^{n}-v_{j-1 / 2}^{n}}{\Delta x}}{\left(\frac{L}{\Delta t}+\frac{R}{2}\right)}
$$

and

$$
v_{j+1 / 2}^{n+1}=\frac{\left(\frac{C}{\Delta t}-\frac{G}{2}\right) v_{j+1 / 2}^{n}-\frac{i_{j+1}^{n+1 / 2}-i_{j}^{n+1 / 2}}{\Delta x}}{\left(\frac{C}{\Delta t}+\frac{G}{2}\right)} .
$$


The sequence of calculations goes as follows. The values $i_{i}^{n+1 / 2}$ and $i_{j}^{n+1 / 2}$ are taken as the driving currents from blocks $D$ and $L$ at $n+1 / 2$. The internal values $i_{j}^{n+1 / 2}$ for $2 \leqslant j \leqslant J-1$ are solved using Eq. (127). The quantities on the right of Eq. (127) are known from previcus calcu'ations. Then Eq. (128) is used to calculate $v_{j+1 / 2}^{n+1}$ for $I \leqslant j \leqslant J-1$. The quantities on the right of Eq. (128) are known from previous calculations. Finally, the voltage at the end points $j=1$ and $j=\mathbf{J}$ is obtained from linear extrapolation and fed back into $D$ and $L$. $D$ and $L$ now take $v_{1}^{n+1}$ and $v_{j}^{n+1}$, and after they are treated by other subroutines, the resulting surrents $i_{1}^{n+1}$ and $i_{j}^{n+1}$ are obtained, thus completing the loop.

In the RAVEN code, $v_{1}^{n+1}$ is inserted in place of the battery voltage in the coupling cell calculation for the field routines.

\section{ACKNOWLEDGMENTS}

The author wishes to express gratitude to J. H. Brownell, I. R. Lindemuth, J. E. Eddleman, and C. Hartman for the helpful discussions, which resulted in the development of the methods used. and to $R$. W. Moses and $\mathbf{L}$. Tumer for enlightening comments on the manuscript. It is a pleasure to acknowledge many stimulating comments from the experimental angle from L. Jones, S. Singer, J. Hammel, J. Schlachter, D. A. Baker, and J. A. Phillips.

\section{REFERENCES}

1. A. Haberstich, "Numerical Calculation of the Theta, Z, and Screw Pinches." Los Alamos National Laboratory report LA-4782-MS (December 1971).

2. D. Steinberg, R. E. Kidder, and A. B. Cecil, "A One-Dimensional Magnetohydrodynamics Code," Lawrence Livermore National Laboratory report UCRL-14931 (1966).

3. T. A. Oliphant, "Dynamic and Quasi-Equilibrium Lagrangian MHD in 1-D," J. Comp. Phys. 38, 4i,j (1980).

4. D. R. Bates, A. E. Kingston, and R. W. P. McWhirter, "Recombination Between Electrons and Atomic Ions I. Optically Thin Plasmas," Proc. Roy. Soc. A257, 297 (1962).

5. J. F. Lee and F. W. Sears, Thermodynamics, Second Ed. (Addison-Wesley Publishing Co., Inc., Reading, Massachusetts, 1963) p. 230.

6. F, H. Harlow and A. A. Amsden, "A Numerical Fluid Dynamics Calculation Method for All Flow Speeds," J. Comp. Phys. 13, 181 (1973).

7. G. Metzger and J.-P. Yabre, Transmission Lines with Pulse Excitation (Academic Press, Inc., New York, 1969) p. 6. 